\title{
Vigilancia tecnológica para la innovación educativa en el uso de bases de datos y plataformas de gestión de aprendizaje en la universidad del Valle, Colombia
}

\section{Technological surveillance for educational innovation in the use of databases and learning management platforms at Universidad del Valle, Colombia}

\author{
Gilbert Andrés Cruz-Rojas ${ }^{1}$ \\ Manuel Alejandro Molina-Blandón ${ }^{2}$ \\ Verónica Valdiri-Vinasco ${ }^{3}$
}

Recibido: agosto 21 de 2018

Aceptado: noviembre 30 de 2018

\section{Resumen}

Este artículo presenta los resultados obtenidos en el primer ciclo del proyecto Vigilancia Tecnológica para la Innovación Educativa, realizado en la Universidad del Valle, con base en una adaptación de la metodología propuesta en la Norma Española Experimental UNE 166006 Gestión de la I+D+i: Sistema de Vigilancia Tecnológica. Este desarrollo metodológico, consistente en tres fases: planeación, monitoreo y construcción de valor, permitió reconocer las dificultades que presentan docentes y estudiantes de la universidad en el uso de bases de datos y plataformas de gestión de aprendizaje, a pesar de su importancia como elementos centrales para fortalecer la producción científica y la calidad educativa. Los resultados de este ciclo permitieron, además, proponer soluciones formativas y tecnológicas que atendieran estas necesidades, buscando mejorar la integración de TIC y fomentar la Innovación Educativa.

Palabras clave: vigilancia tecnológica, innovación educativa, educación superior, tecnología de la información.

\begin{abstract}
This article presents the results obtained in the first cycle of the Technological Surveillance project for Educational Innovation, carried out at the Universidad del Valle, based on an adaptation of the methodology proposed in the Spanish Experimental Standard UNE 166006 R + D + i Management: Technological Surveillance System. This methodological development, consisting of three phases: planning, monitoring and value construction, allowed to recognize the difficulties that teachers and students of the university have in the use of databases and learning management platforms, despite their importance as elements central to strengthen scientific production and educational quality. The results of this cycle allowed, in addition, to propose training and technological solutions that meet these needs, seeking to improve the integration of ICT and promote Educational Innovation.
\end{abstract}

Keywords: technological surveillance, educational innovation, higher education, information technology.

1 Magíster en Educación, Universidad del Valle, Cali, Colombia. E-mail: gilbert.a.cruz.r@correounivalle.edu.co

2 Licenciado en Literatura, Magíster en Comunicación, Universidad del Valle, Cali, Colombia. E-mail: manuel.a.molina@correounivalle. edu.co

3 Licenciada en Lenguas Extranjeras, Magíster en Dirección y Producción de e-Learning, Universidad del Valle, Cali, Colombia. E-mail: veronica.valdiri@correounivalle.edu.co 


\section{Introducción}

La Vigilancia Tecnológica (VT) surge de la necesidad de las organizaciones de obtener información útil para generar conocimiento, cumplir sus objetivos y anticiparse a sus competidores, a partir de un análisis interno y externo. En el campo empresarial, la VT suele articularse con procesos de Inteligencia Competitiva y determina aspectos claves para fortalecer la Investigación, Desarrollo e Innovación (I+D+i). Emplear la VT en el campo de la educación superior es un método innovador pero complejo, ya que, se trata de la adopción de un sistema creado para un mundo organizacional en constante competencia. Con la VT se busca tomar decisiones de manera ágil, eficiente y segura al menor costo posible y de esta manera ofrecer productos y servicios de calidad previendo las posibles barreras de entrada para su comercialización, además de que permite conocer el estado del mercado global (Bollás \& Valencia, 2017).

Este documento se deriva de la investigación desarrollada en el marco del proyecto de Vigilancia Tecnológica (VT) para la Innovación Educativa, el cual, fue planteado una iniciativa para conocer las necesidades académicas y tecnológicas de docentes y estudiantes de la Universidad del Valle, para proponer después posibles soluciones por medio de la oferta de productos y servicios. El estudio permitió conocer las necesidades académicas de algunos profesores y estudiantes y proponer soluciones, relacionadas con las tendencias nacionales y globales en integración de tecnologías en las prácticas educativas.

En este artículo se presentan los resultados de un ciclo de Vigilancia Tecnológica realizado en la Universidad del Valle, mediante una adaptación de la Metodología de VT de la norma UNE 166006:2011 (Asociación Española de Normalización \& Certificación, 2011). En el desarrollo del ciclo se considera que la educación universitaria, como formadora de las nuevas generaciones de profesionales, debe atravesar continuamente procesos de innovación educativa que le permitan mantenerse al día y asegurar buenos niveles de calidad en su formación, al tiempo que promueve cambios sociales. Parte de estos procesos tienen que ver con la integración y apropiación en el uso de tecnología por parte de profesores y estudiantes de la institución. La Universidad del Valle reconoce las TIC como un elemento diferenciador para fortalecer sus gestiones académicas e investigativas a través de ambientes de aprendizaje mediados por las mismas.

El artículo se estructura en cinco secciones que dan respuesta a los siguientes interrogantes: ¿cuál es el marco de referencia?, ¿cuál fue el diseño metodológico?, ¿cuáles fueron los principales hallazgos? y ¿qué conclusiones emergen a partir del estudio?.

\section{Marco de referencia y metodología}

\subsection{Innovación educativa}

Caracterizar prácticas asociadas a la integración de TIC en un contexto educativo específico es un proceso complejo, que requiere una revisión permanente de conceptos y fuentes de información. Considerando que el objetivo de este trabajo se relaciona con el concepto de Innovación Educativa (IE), se ha tenido en cuenta la propuesta realizada por el Ministerio de Educación Nacional (2016) y la Universidad del Valle, en el proyecto Observatorio Colombiano de Innovación Educativa con uso de $\mathrm{TIC}$, donde se indica que:

(...) La Innovación Educativa se configura a partir de la creación o apropiación de nuevas ideas, estrategias, procesos, conocimientos, metodologías o productos, que generan transformaciones en las dinámicas de la comunidad educativa y la cultura escolar (...) Al ser una innovación, se puede observar, es deliberada, apropiada por los actores involucrados, adaptable para otros y no ocurre con frecuencia. (p. 5) 
No obstante, para enfocarse mejor en el contexto de la educación superior, se consideraron también los planteamientos de Casas y Stojanovic (2013), quienes definen la innovación universitaria como el proceso de transformación de las universidades en instrumentos que promuevan los grandes cambios sociales y científicos necesarios para la llamada sociedad del conocimiento (Castells, 2002). Según Casas y Stojanovic (2013), dicha transformación no se limita a reemplazar una práctica por otra sino a mejorar, reformar y alcanzar resultados institucionales más efectivos, conjugando labores científicas y tecnológicas con otras de tipo organizacional, financiero y comercial. Esto con el fin de modificar las estructuras generales y productivas de cualquier sociedad contemporánea.

Este proyecto funcionó, entonces, como una apuesta para generar transformaciones que lograran ser apropiadas por la comunidad universitaria, produciendo mejoras en los procesos de enseñanza y aprendizaje, transformaciones que tienen que ver con integración de tecnologías digitales en las prácticas educativas, pero que son acordes a las necesidades de los actores de un contexto determinado, pues como plantea Salinas (2004): "ninguna innovación puede ignorar el contexto en el que se va a desarrollar. La introducción de las TIC en la docencia universitaria supone considerar aspectos que hacen referencia a las características, tanto individuales como colectivas, de los posibles usuarios" (p. 9). Estas innovaciones deben, además, impactar de forma integral todos los elementos intrínsecos de la comunidad universitaria, tal como lo proponen Bautista, Borges y Forés (2006):

(...) Innovar en educación superior es poner en juego toda la red de elementos y sistemas que forman parte de la comunidad educativa: los estudiantes, los docentes, los materiales, los contextos educativos, los requisitos tecnológicos o institucionales, etc. (...) Los procesos de innovación pedagógica deben ir acompañados de un apoyo institucional y un convencimiento general de la necesidad de una mejora continua de la educación. (p.189)
De acuerdo a lo anterior, se tiene que el objetivo final de un proyecto enfocado en la IE debe ser, como señalan Gros y Lara (2009), posicionar a la universidad como institución generadora de conocimiento, la cual se transforma y mejora continuamente por medio de la implementación de productos y servicios tecnológicos probados y documentados: "Los resultados de innovación obtenidos a través de los proyectos estratégicos se traducen en productos entendidos como instrumentos, metodologías o recursos para la formación. Estos han de ser probados y analizados antes de consolidarse como productos." (p. 239). Según Salmon (2010), es clave que la universidad defina qué tecnologías de aprendizaje son prioritarias para garantizar la innovación, para así promoverlas y financiarlas institucionalmente. Esto sin dejar a un lado las tecnologías periféricas, que pueden ser incorporadas por estudiantes y profesores de manera aislada, pero que también generan innovación.

Finalmente, resulta importante considerar la perspectiva de Ferrés, Masanet y Mateus (2018) quienes, basados en Johnson (2010), señalan la necesidad de crear un entorno donde se propicie la innovación, por medio de la exploración y combinación de las relaciones entre personas y materiales, entorno que a veces no se da en el sistema universitario debido a su rigidez, cultura del control vertical y transmisión del conocimiento, en las cuales se privilegian productos por encima de procesos. Por su parte, Bates (2010) argumenta que la misión principal de la universidad debe mantenerse pero es inevitable un cambio radical en su organización y gobernanza, lo que le permitiría adaptarse a las necesidades del siglo XXI. De igual manera, sostiene que este cambio es mucho más lento en las universidades públicas, tal y como sucede en la Universidad del Valle, donde los procesos de innovación y de integración de TIC en las prácticas educativas o el de virtualización de programas académicos, aún son incipientes. 


\subsection{Vigilancia tecnológica}

Moya-Espinosa y Moscoso-Durán (2017) sostienen que la Vigilancia Tecnológica, y la Inteligencia Competitiva, son dos herramientas complementarias y útiles para anticipar un suceso y mejorar la competitividad de una organización. No obstante, aclaran que:

(...) El ejercicio de la vigilancia tecnológica se encuentra más directamente vinculado con la captación y análisis intrínsecos de las informaciones, mientras que el desarrollo de la inteligencia competitiva está orientada hacia la interpretación de esas informaciones previamente seleccionadas para ayudar a la toma de decisiones. (p. 20)

En este sentido, es importante considerar las normas que la entidad normalizadora y certificadora AENOR ha publicado, dada la importancia de la I+D+i en el progreso económico y social (Aldasoro-Alustiza, Cantonner-Jordi \& Cilleruelo-Carrasco, 2012). En la Norma Española Experimental UNE 166006 Gestión de la I+D+i: Sistema de Vigilancia tecnológica (Asociación Española de Normalización y Certificación, 2011) se define la VT como un:

(...) Proceso organizado, selectivo y permanente, de captar información del exterior y de la propia organización sobre ciencia y tecnología, seleccionarla, analizarla, difundirla y comunicarla, para convertirla en conocimiento para tomar decisiones con menor riesgo y poder anticiparse a los cambios.

Por su parte, Marulanda, Hernández y López (2016), basados en Izarral, Sánchez y Caira (2014), plantean que la VT:

(...) Contempla los avances científicos y técnicos, fruto de la investigación básica y aplicada, los productos y servicios, los procesos de fabricación, los materiales, su cadena de transformación, las tecnologías y sistemas de información. Se encargan de analizar las tecnologías disponibles o que acaban de aparecer y capaces de intervenir en nuevos productos o procesos. (p. 19)

De acuerdo a lo anterior, se consideró la VT como un método pertinente para promover la innovación en la Universidad del Valle pues, a partir del análisis de diferentes prácticas, es posible obtener información útil para solucionar necesidades concretas, por medio del desarrollo de productos y servicios tecnológicos. Al mismo tiempo, la VT facilita la recopilación, sistematización y divulgación de información selectiva que le permite a diferentes grupos de usuarios, con intereses relacionados, conocer los diferentes procesos y avances que se llevan a cabo en otras áreas. En el campo de la educación superior, la información obtenida a través de ciclos de VT le permite a las instituciones educativas tomar decisiones sobre sus procesos de gestión del conocimiento, especialmente los de formación, y mejorarlos por medio de soluciones innovadoras y eficaces.

Se reconoce la existencia de varios modelos de VT, para el desarrollo de diferentes tipos de investigación, como los que reseñan Bollás y Valencia (2017), destacando propuestas como la norma UNE 166006 de 2006, la metodología Quicklook para la comercialización de tecnología, la metodología de Vigilancia Tecnológica e Inteligencia Competitiva por Sánchez y Palop, entre otras. En educación superior, varios de estos modelos sugieren la inclusión y participación de la mayor parte de los miembros de la comunidad universitaria dentro de los estudios realizados. Por su parte, Marulanda, et al. (2016), resaltan la importancia del involucramiento de los estudiantes, pues son éstos quienes deben sacar el máximo provecho de las herramientas y recursos ofrecidos por las universidades.

Sin embargo, reconocer la tensión que se produce al utilizar un método propio de la ingeniería y las ciencias empresariales para promover la innovación en una institución educativa, cuyo fin no es 
precisamente utilitario, es un reto para los diferentes actores que participan en un proceso de VT, en donde son necesarias adaptaciones que consideren la complejidad del campo educativo así como una revisión de diferentes experiencias que hayan empleado sistemas de VT en la educación superior.

\subsection{Formación docente en educación superior}

Otro referente clave en la consolidación de este proyecto es el concepto de formación en educación superior, el cual tiene particularidades que lo diferencian de otros contextos educativos. Una de estas particularidades se asocia con la formación docente, pues se ha identificado que el profesorado universitario, en la mayoría de casos, no ha sido formado para ejercer la docencia, lo cual genera dificultades en las prácticas que desarrolla. Según Torello (2011), esto ocurre porque el profesor no ha tenido la necesidad de formarse en estos asuntos y la Institución tampoco se lo ha exigido. En ese sentido, parece necesaria una transformación en la que se reconozca la importancia que tiene la formación en la docencia universitaria pues es "una labor intelectual que requiere un reposicionamiento de la misma como actividad creativa y rigurosa, desde los mismos profesores que la emprenden hasta las instituciones que la apoyan" y que "se convierte, también, en un terreno fértil para la generación de conocimiento sobre los propósitos, estrategias y efectividad de los procesos de enseñanza y aprendizaje" (Ferro, 2014, p. 84).

En consecuencia, es clave reconocer que la docencia es una de las funciones profesionales de los profesores universitarios, al igual que la investigación. Torello (2011) resalta que en estas funciones profesionales se debe distinguir el impacto según los contextos en los cuales las prácticas se dan y considera fundamental determinar el contexto social, institucional y el microcontexto. Sin embargo, Villegas (2010) resalta que "La transformación en busca de mejorar la calidad en la educación, independientemente del nivel educativo, debe incluir la mejora del profesorado no sólo en la especialidad disciplinar y en destrezas y habilidades docentes, sino también en otros aspectos" (p. 95).

Dichos aspectos están relacionados con el uso e integración de Tecnología Digital en las prácticas educativas y pedagógicas de los docentes, así como sus diferentes variables. Por su parte, Salinas (2000), citado en Villegas (2010), considera que el reto en mención debe tener en cuenta las implicaciones que tienen las TIC para el desarrollo de nuevas habilidades en los docentes, por lo menos en tres ámbitos: a) las TIC en el salón de clase tradicional, b) en las modalidades no convencionales y c) el uso doméstico de las TIC.

\subsection{Metodología}

Con el fin de ofrecer a la comunidad académica de la Universidad del Valle productos y servicios tecnológicos pertinentes para favorecer la IE en todas las modalidades de formación, como se mencionó, se realizó una adaptación de la Metodología de VT de la norma UNE 166006:2011 (Asociación Española de Normalización \& Certificación, 2011). Se definieron tres fases metodológicas (planeación, monitores y construcción de valor), las cuales, fueron implementadas por dos vigías tecnológicos, asesorados por un equipo interdisciplinario de apoyo. Algunas de las acciones llevadas a cabo en cada fase se presentan en la tabla 1. 
Tabla 1. Acciones realizadas en el primer ciclo de Vigilancia Tecnológica.

\begin{tabular}{|c|c|c|}
\hline Fase & Etapa & Acciones \\
\hline \multirow[t]{9}{*}{ Planeación } & Formulación & - Identificar unidades académicas. \\
\hline & & - Definir fuentes de información. \\
\hline & & - Determinar un plan de muestreo. \\
\hline & Trabajo de Campo & - Diseñar y realizar entrevistas. \\
\hline & & - Transcribir las entrevistas. \\
\hline & & - Consolidar notas sobre el trabajo. \\
\hline & $\begin{array}{l}\text { Análisis y construcción de } \\
\text { fichas }\end{array}$ & - Revisar la información de la etapa anterior. \\
\hline & & - Estudiar y priorizar necesidades. \\
\hline & & - Elaborar fichas de identificación \\
\hline \multirow[t]{5}{*}{ Monitoreo } & Búsqueda de información & - Recopilar y clasificar la información bibliográfica. \\
\hline & & - Profundizar en las fuentes seleccionadas. \\
\hline & & - Diseñar y realizar encuestas. \\
\hline & $\begin{array}{l}\text { Tratamiento y profundiza- } \\
\text { ción de la información }\end{array}$ & - Documentar las necesidades. \\
\hline & & - Autoevaluar y validar propuesta de soluciones. \\
\hline \multirow[t]{2}{*}{$\begin{array}{l}\text { Construcción } \\
\text { de valor }\end{array}$} & $\begin{array}{l}\text { Diseño de soluciones tec- } \\
\text { nológicas }\end{array}$ & $\begin{array}{l}\text { - Determinar objetivos, productos y actividades de } \\
\text { acuerdo a cada propuesta de solución. }\end{array}$ \\
\hline & $\begin{array}{l}\text { Diseño y desarrollo de } \\
\text { estrategias y acompaña- } \\
\text { miento }\end{array}$ & $\begin{array}{l}\text { - Establecer una propuesta de desarrollo para la for- } \\
\text { mulación de las actividades. }\end{array}$ \\
\hline
\end{tabular}

En el desarrollo de cada fase se utilizaron diferentes tipos de instrumentos para la recolección de información, dentro de los que se destaca una entrevista de fuentes para identificación de necesidades, fichas de identificación y fuentes y ficha de identificación de soluciones tecnológicas.

\section{Resultados y discusión}

Al culminar la fase de planeación, se identificaron veinte necesidades en las dos unidades académicas intervenidas (Ver tabla 2). 
Tabla 2. Necesidades identificadas durante la primera fase.

\begin{tabular}{|c|c|}
\hline & Necesidades \\
\hline 1 & Falta de cultura de uso del Campus Virtual \\
\hline 2 & Falta de infraestructura tecnológica en los salones de clase \\
\hline 3 & Oferta programas de formación virtual con el respaldo de la universidad para público en general \\
\hline 4 & $\begin{array}{l}\text { Más formación en el uso de bases de datos y demás recursos de investigación, ofrecidos por la biblioteca } \\
\text { para docentes y estudiantes }\end{array}$ \\
\hline 5 & Garantizar el uso adecuado de plataformas para el aprendizaje de idiomas en los cursos de segunda lengua \\
\hline 6 & Falta de formación en derechos de autor y herramientas antiplagio \\
\hline 7 & Mejorar habilidades de aprendizaje autónomo y trabajo independiente de los estudiantes \\
\hline 8 & Promover y socializar las políticas de innovación educativa definidas para la universidad \\
\hline 9 & Formación para docentes en creación de productos audiovisuales \\
\hline 10 & Mejorar el uso del correo institucional \\
\hline 11 & $\begin{array}{l}\text { Fortalecimiento del uso de videoconferencia para contactarse con pares académicos y mejorar integración } \\
\text { académica }\end{array}$ \\
\hline 12 & Herramientas para reforzar procesos de escritura - actividades de escritura potenciadas con tecnología \\
\hline 13 & $\begin{array}{l}\text { Sobrepoblación en los cursos que imposibilitan una intervención más fuerte en procesos de escritura y } \\
\text { lectura }\end{array}$ \\
\hline 14 & Falta de un repositorio de recursos audiovisuales libres para las diferentes áreas del conocimiento \\
\hline 15 & Falta de laboratorios de idiomas \\
\hline 16 & Formación para hacer presentaciones presenciales-virtuales \\
\hline 17 & Formación en el uso de redes sociales para la formación. \\
\hline 18 & Formación en uso adecuado de redes sociales en el ambiente universitario. \\
\hline 19 & Falta de talleres virtuales o centros de escritura \\
\hline 20 & Acompañamiento a las licenciaturas para cursos en formación de TIC en educación \\
\hline
\end{tabular}

De estas necesidades, siete $(1,2,4,5,8,12,14)$, se documentaron dentro de las fichas de necesidades y fuentes, según la recurrencia con la que fueron mencionadas en las entrevistas y el impacto para la comunidad educativa. Para la segunda fase, se seleccionaron cinco $(1,4,5,8,14)$, y se analizaron en procura de plantear posibles soluciones. Finalmente, considerando los hallazgos y los alcances del proyecto, se escogieron sólo dos necesidades ( 1 y 4), a las cuales se les hizo un análisis exhaustivo seguido de una propuesta de solución pertinente y justificada. El desarrollo del ciclo realizado se presenta en la tabla 3. 
Tabla 3. Desarrollo del ciclo de Vigilancia Tecnológica.

\begin{tabular}{ll}
\hline Fase & Desarrollo \\
\hline & La Escuela de Estudios Literarios y Escuela de Ciencias del Lenguaje fueron las unidades académi- \\
& cas seleccionadas para la intervención. Estas unidades fueron escogidas por la afinidad disciplinar \\
& con el perfil profesional de los vigías encargados de realizar el proceso. Además, se definieron las \\
& fuentes de información (directivos de las Escuelas y docentes) y se realizó un plan de muestreo no \\
planeación & probabilístico por conveniencia, pues se seleccionaron individuos a los que se tuvo un fácil acce- \\
& so. En este proceso se logró entrevistar a 11 docentes usando como instrumento metodológico la \\
& entrevista semi-estructurada que, en este caso, permite adentrarse en la realidad de los sujetos y \\
& conocer las necesidades identificadas en sus quehaceres
\end{tabular}

A partir del trabajo de campo y análisis realizado se elaboraron fichas de identificación y fuentes

Monitoreo Se revisaron las necesidades priorizadas a través de un instrumento de evaluación que establecía escalas para medir el impacto, la dificultad de implementación y la importancia dentro del Plan Estratégico 2015 de la Universidad del Valle. A partir de los puntos obtenidos, se determinaron cinco de siete necesidades que debían continuar en esta fase de monitoreo, en la que se buscaba profundizar en algunos elementos determinados en las fichas de identificación. Para esta fase se propusieron dos etapas:

En la búsqueda de información se recopiló y clasificó la información obtenida, lo que implicó profundizar en las fuentes identificadas a través de una encuesta en línea, realizada con un muestreo probabilístico aleatorio simple (MAS), en el cual se seleccionaron de manera aleatoria usuarios inscritos en el campus virtual y pertenecientes a las dos escuelas escogidas. El tamaño de muestra calculado fue de 105 usuarios (docentes y estudiantes), sin embargo, en este estudio se obtuvieron datos de 138 usuarios. Para llegar a las unidades de muestreo se realizaron encuestas a través de correo electrónico, con preguntas tanto abiertas como cerradas y las variables que se analizaron fueron principalmente cualitativas.

Para el tratamiento de información: se documentaron las necesidades establecidas realizando análisis de la situación actual de la necesidad (caracterizaciones y evaluaciones de uso y desempeño de las herramientas mencionadas e iniciativas anteriores de solución como programas de formación realizados). Luego se hizo un ejercicio de autoevaluación para proponer y validar las soluciones más pertinentes y viables, de acuerdo a la información disponible.

Construcción de valor
En la construcción de valor se retomaron dos de las propuestas de solución escogidas en la fase anterior, para precisar algunos objetivos y plan de acción a desarrollar.

A continuación, se exponen brevemente las tres necesidades que se trabajaron en las fases de planeación y monitoreo pero que fueron descartadas en la fase de construcción de valor, seguidas de las dos necesidades que pasaron a la fase final.

\subsection{Plataformas para aprendizaje de Segunda Lengua}

Como parte de las estrategias de trabajo autónomo de los estudiantes, la Escuela de Ciencias del Lenguaje ha adquirido diferentes softwares (Tell me more; Clarity English; Dexway) que facilitan el acceso a recur- 
sos y actividades de calidad para los estudiantes de la Licenciatura en Lenguas Extranjeras y los estudiantes de los cursos de inglés con fines generales y académicos (EGAP). No obstante, el uso de estos softwares es bastante limitado por parte de los estudiantes, lo que hace que el recurso esté subutilizado.

La importancia de estos softwares se debe a que el aprendizaje autónomo en lengua extranjera es uno de los determinantes para alcanzar niveles altos de proficiencia. Los aprendices de lengua extranjera deben desarrollar sus propias estrategias de aprendizaje, dado el poco tiempo que tienen dentro del salón de clases con sus profesores (Harmer, 2003). Como lo expresa Muchlis (2015), "cuando los estudiantes se dan cuenta de que el éxito de su aprendizaje depende tanto de ellos como de sus docentes, empiezan a tener autonomía, sin esperar que les digan qué hacer" (p.276, traducción propia). De acuerdo con esto, el éxito en el aprendizaje de lengua extranjera depende de la proactividad del estudiante y del tiempo de exposición y práctica significativa que este tenga fuera del salón de clases, en espacios en los que pueda atender a sus necesidades personales y trabajar en sus dificultades. En este sentido, la tecnología es una gran aliada, especialmente en contextos en los que la L2 es una lengua extranjera, y por tanto no es fácil exponerse a contextos auténticos de uso.

3.2 Repositorios virtuales para enseñanza de segunda lengua

La falta de repositorios virtuales para la enseñanza de una segunda lengua fue otra de las problemáticas encontradas en la investigación. Dentro de los procesos de aprendizaje de una lengua extranjera, la exposición a documentos orales y visuales (grabaciones de audio y video) en la lengua meta es fundamental, especialmente si son documentos auténticos, es decir, aquellos que no fueron creados con propósitos instruccionales. A través de estos documentos, los aprendices se enfrentan a la lengua en uso real, a situaciones en las que la comunicación ocurre en contextos naturales y coti- dianos para los nativos de la misma. Es sabido que la habilidad de comprender materiales orales es una de las más difíciles de desarrollar debido a las diferentes variables como el tono, la entonación, el acento, el vocabulario empleado, desconocimiento del principio y/o la continuación de la conversación, incapacidad para separar la información en unidades más pequeñas y problemas de concentración (Vandergrift, 2007; Aldera, 2015). A estas variables se le suma la poca exposición que tienen los aprendices al mismo, pues la mayor parte de su exposición oral a la lengua viene de parte del profesor.

La búsqueda de información detectó que los profesores de la universidad requieren de grandes cantidades de tiempo para la selección de material para la comprensión oral pertinente a sus cursos. La mayoría de docentes reportan que las mayores dificultades que enfrentan a la hora de seleccionar el material es encontrar grabaciones cuyo grado de dificultad se ajuste al nivel de los estudiantes y que, de preferencia, sea material auténtico. Adicionalmente, los docentes de lenguas extranjeras consideran importante tener en cuenta las necesidades de formación de los estudiantes y sus intereses académicos de acuerdo a los programas académicos a los que pertenecen.

3.3 Socialización de las políticas de innovación educativa de la universidad

Las entrevistas realizadas en la primera fase evidenciaron que los docentes seleccionados tienen diferentes visiones del término IE: pedagógicas, curriculares, tecnológicas. Además, desconocen las iniciativas que se han venido realizando en la universidad para promover la innovación educativa con uso de TIC. Desconocimiento que se justifica al encontrar que la Universidad no tiene como tal una política de IE definida. En el Plan de Incorporación de TIC a los procesos educativos 20122025, se propone como visión que: "la Universidad del Valle será reconocida regional, nacional e internacionalmente por su alto nivel de innovación en la incorporación de las TIC en la docencia, la in- 
vestigación y la extensión" (Universidad del Valle, 2012, p.16).

Pese a esto, no hay información sobre el avance de las acciones planteadas en el documento; por lo cual se hace necesario el diseño de esta política acompañada por un proceso de socialización con toda la comunidad educativa, a través de campañas de gestión de cambio y programas de formación. Como dice Salinas (2004), “Es importante que el proyecto de innovación esté integrado en la estrategia institucional y que la comunidad universitaria lo asuma. Se supone que todos los miembros de la comunidad (dirección, profesorado, etc.) deben mostrar compromiso con el proyecto." (p.10). Con este mismo propósito, es importante identificar los medios de difusión adecuados y a aquellos docentes que puedan impulsar, e incluso proponer proyectos de innovación y ejerzan influencia sobre aquellos que son más resistentes al cambio y difíciles de persuadir para adoptar innovaciones (Rogers, 1995).

\subsection{Uso de bases de datos académicas}

Al 2016, la División de Bibliotecas de la Universidad del Valle cuenta con 127 bases de datos académicas que incluyen un total de 223.320 libros electrónicos y 43.155 revistas electrónicas. Estos pueden ser consultados en cualquier equipo conectado a la Intranet de la Universidad del Valle o por fuera de la universidad a través de la clave de acceso remoto. Sin embargo, los resultados de la primera fase evidenciaron un bajo uso de estos recursos por parte de estudiantes y docentes de las Escuelas de Estudios Literarios y Ciencias del Lenguaje. Los docentes entrevistados manifestaron que los estudiantes hacen poco uso de ellas y que su acceso no está garantizado desde dentro o fuera de la universidad. Además, se detectó que las búsquedas de los estudiantes son básicas y se restringen a lo que encuentran en Internet, lo cual afecta la calidad de los trabajos de curso e incluso de los trabajos de grado. Esto coincide con lo planteado por Matus, Menay, Loyola y Cordeiro (2009):
(...) Se puede señalar que debido a los cambios culturales y al acceso tecnológico, los estudiantes universitarios se definen como "conocedores y en ocasiones expertos en la búsqueda de información en línea", situación que llevada a la realidad se traduce en un manejo avanzado en servicios de chat, mensajería y/o programas para descargar música o videos. Frente a tal escenario se hace primordial señalar que requieren ser capacitados, pues la búsqueda de información no se limita a un copiar y pegar los resultados obtenidos a través de búsquedas básicas que, por lo demás, muchas veces son documentos que no involucran calidad ni rigor científico. (p. 5)

Adicionalmente, se encontró que los docentes tampoco muestran un uso significativo de las bases de datos. La mayoría de los docentes entrevistados reconoce no tener un dominio que les permita usar estos recursos asiduamente ni mucho menos promover su uso entre los estudiantes. Infante-Moro, Torres, Infante-Moro y Muñoz (2014), explican que:

(...) Es cierto que el uso de las bases de datos es poco frecuente en los docentes, las suelen utilizar para la elaboración de sus tesis doctorales o si el docente está participando en una investigación; se hace poco uso para la docencia $y$, en realidad, la ciencia está en estas revistas científicas recogidas en ellas. A pesar de ello, sí que existe obsesión por la publicación de artículos JCR porque es lo que la ANECA valora a los docentes, pero cuando se recurre a la búsqueda de información para la docencia resulta que no se acude a estas bases de datos científicas. (p. 7)

Considerando lo anterior, se propuso potenciar, en estudiantes y docentes de las escuelas mencionadas, el uso de los recursos electrónicos de investigación disponibles. Se avanzó, entonces, en la identificación de necesidades concretas de formación así como también de elementos que inciden en el uso y en la oferta de formación existente. 
En lo referente a la oferta de formación, se encontró que existen capacitaciones sobre los diversos servicios y recursos de la biblioteca pero ninguna de ellas focalizada en las necesidades de las unidades académicas, por tanto aún no se logra comprender el impacto que pueden tener estos recursos en las labores de docencia e investigación. La mayoría de las capacitaciones que se realizan actualmente no cuentan con el tiempo necesario para que los usuarios puedan profundizar en las potencialidades investigativas de las distintas herramientas. Tampoco se da la posibilidad de que los usuarios puedan realizar ejercicios prácticos mientras el capacitador explica los diferentes temas. A excepción de algunos talleres especializados, donde se trabajan temas exclusivos de salud y medicina, todas las capacitaciones se hacen sin modelar tareas que el usuario pueda emplear en el quehacer investigativo de su área. Tampoco se trabajan actividades para que los docentes promuevan el uso de estos recursos en el aula de clase.

En la encuesta de muestreo en línea realizada a docentes y estudiantes, se logró evidenciar que existe un gran desconocimiento de las potencialidades de las bases de datos. Ambos tipos de usuarios presentan concepciones equivocadas sobre lo que se puede hacer con estos recursos. Esto, sumado a la falta de instrucción y acompañamiento en el acceso a ellos, produce una desmotivación y un bajo índice de uso. Por consiguiente, se hace necesaria la implementación de programas de formación acordes a las necesidades concretas de los docentes, preferiblemente relacionados con sus áreas de interés académico. Esto en procura de mejorar la participación en dichos programas, sumado a una estrecha coordinación con las unidades académicas para incentivar la asistencia de los docentes. Adicional a esto, se sugiere que estos programas incluyan acciones de acompañamiento posteriores; ello podría garantizar la implementación de los aprendizajes en el aula de clase.

\subsection{Uso de Campus Virtual Univalle}

En la primera fase del ciclo de VT también se detectó la necesidad de formar a los docentes y estudiantes en un mejor uso y aprovechamiento del Campus Virtual (Plataforma LMS Moodle de Univalle). Un $45 \%$ de los docentes entrevistados reconocieron no usar el Campus, mientras otros declararon un uso muy básico del mismo. De igual manera, la encuesta de muestreo en línea realizada a usuarios del Campus Virtual de las dos escuelas, confirmó que las herramientas más usadas son subir archivos y tarea. A través de esta misma encuesta se confirmó la poca participación de los docentes en los talleres de capacitación sobre Campus Virtual que ofrece la universidad. Respecto a los estudiantes, se encontró que un $59 \%$ de encuestados cree que los docentes conocen el Campus pero no lo dominan y un $60 \%$ admite que desearía participar en programas de formación para un mejor uso de esta plataforma.

En la fase de monitoreo, se analizó y caracterizó la oferta de formación que DINTEV ha puesto en marcha para promover el uso del Campus Virtual. Se encontró que desde el segundo semestre del 2005 al primer semestre de 2017, se realizaron 271 talleres de capacitación, a los cuales asistieron 2049 usuarios entre docentes y estudiantes. Estos talleres, de duración promedio de 4 horas, tienen un nivel básico en el que se explica la configuración inicial del curso en Moodle y las herramientas de publicación (archivo, carpeta, etiqueta, enlace); y un nivel complementario, donde se trabajan las herramientas de comunicación y evaluación (foro, chat, cuestionario). El análisis permitió determinar que la mayoría de los docentes sólo asisten al taller básico, lo que podría explicar sus limitaciones de uso.

Dado que 4 horas de taller resultan insuficientes para explorar todas las herramientas y recursos de Moodle, se hizo necesario implementar un curso de mayor intensidad denominado Explorando el diseño y la configuración de las actividades de aprendizaje en el Campus Virtual. En este progra- 
ma, se propone al docente crear y modelar un curso a la medida de sus necesidades, partiendo de los usos didácticos que tiene cada herramienta o recurso.

A pesar de estos esfuerzos de formación, los resultados de la primera fase del ciclo de VT mostraron la necesidad de implementar mejoras que incentiven un uso más significativo del Campus Virtual y que se encaminen hacia nuevas metodologías de formación, como el aula invertida, los cursos mixtos (virtual-presencial) y los cursos virtuales, y que, de paso, estén alineados con las nuevas propuestas de formación de la Universidad. Esto es prioritario de cara a la innovación educativa, pues una buena disposición de los docentes para utilizar las plataformas LMS puede impulsar un uso más significativo entre los estudiantes. Además, una mejor utilización de las mismas permite potenciar los resultados académicos de la institución, como está documentado en varios estudios (Baker, Rodríguez-Duarte \& Sandulli, 2014).

En una segunda parte del análisis, se realizó una evaluación de cursos creados en la plataforma por docentes participantes en el programa de formación Explorando el diseño y la configuración de las actividades de aprendizaje en el Campus Virtual, los cuales pertenecen a distintas unidades académicas de la universidad. Los resultados permitieron concluir que la gran mayoría aún tiene deficiencias en los tres componentes propuestos (apariencia y funcionalidad del curso, aspectos pedagógicos y didácticos, uso de herramientas y recursos). Muchos no cumplen los criterios evaluados y sólo logran subir gran cantidad de archivos, publicar contenidos para todo el semestre e incluir algunas herramientas de evaluación. Hay grandes falencias en el componente de aspectos pedagógicos y didácticos, pues la mayoría de cursos revisados no contienen actividades de aprendizaje con objetivos claros, para promover la interacción y el uso de los materiales disponibles. Finalmente, se destaca también que los docentes utilizan un número limitado de herramientas y recursos, a pe- sar de la gran cantidad de posibilidades disponibles en Moodle.

Simultáneamente, se encontró que en las escuelas seleccionadas también hay experiencias destacadas de uso del Campus Virtual, las cuales fueron sometidas a evaluación a través del mismo instrumento con el que se evaluaron los docentes participantes del curso de formación. A diferencia del primer grupo, muchos de estos docentes sí cumplen con los criterios planteados en los componentes de apariencia y uso de herramientas y recursos. La mayoría logra crear cursos con apariencia atractiva y con actividades que casi siempre son resueltas por los estudiantes en la misma plataforma. Además, utilizan recursos multimedia que combinan diferentes tipos de información, y emplean más herramientas que los docentes del primer grupo. Sin embargo, en el componente de aspectos pedagógicos y didácticos, comparten falencias en la creación de actividades de aprendizaje, las cuales carecen de objetivos claros y de interacción con y entre estudiantes. Sólo logran cumplir en el uso de herramientas de evaluación y en fomentar el uso del material disponible para realizar las tareas planteadas.

Al igual que en la necesidad anterior, estos hallazgos llevan a pensar que es necesario poner en marcha programas de formación que respondan a las necesidades concretas de los docentes y que se relacionen con sus áreas de interés académico. Esto complementado con estrategias de acompañamiento que apoyen la implementación de lo aprendido, tal como ya mencionamos anteriormente.

Siguiendo estas consideraciones, el equipo de vigías tecnológicos del proyecto logró consolidar documentación que aporta a la posible solución de las problemáticas surgidas en las dos necesidades seleccionadas.

En lo relacionado con la necesidad de potenciar el uso de los recursos electrónicos disponibles en 
las bases de datos académicas de la Biblioteca se logró.

i. Caracterizar la oferta de formación existente y analizar el uso de las bases de datos académicas en las dos escuelas seleccionadas.

ii. Diseñar e implementar un programa de formación en desarrollo de habilidades informacionales para usar y promover las bases de datos académicas adquiridas por la Universidad del Valle.

iii. Crear y publicar una clasificación de bases de datos con contenidos para la enseñanza y aprendizaje de las humanidades y disciplinas afines.

Por otro lado, en lo concerniente a la necesidad de robustecer el uso pedagógico y didáctico del Campus Virtual, el equipo de vigías logró:

i. Caracterizar la oferta de formación existente y analizar el uso del Campus Virtual de la Universidad del Valle en las dos escuelas seleccionadas.

ii. Diseñar e implementar un programa de formación en Campus Virtual enfocado en actividades de lectura y escritura.

iii. Realizar mejoras y actualizaciones en la interfaz del Campus Virtual y promover su divulgación.

\section{Conclusiones}

Las necesidades encontradas a través de este ciclo de VT, revelan que las propuestas de mejoramiento que tengan que ver con el uso de servicios tecnológicos ya existentes, como son bases de datos y Campus Virtual, deben contar con propuestas de formación docente enfocadas hacia los usos pedagógicos de las herramientas, y con acompañamiento durante las etapas de implementación, como componentes fundamentales para asegurar el éxito de las mismas. Para ambos casos, aunque la Universidad ya cuenta con procesos de formación periódicos, no se implementan planes de acompañamiento que garanticen que se ponga en práctica lo aprendido, lo cual tiene como resultado el uso poco frecuente o ineficiente de ambas herramientas.

La difusión de los hallazgos y de las propuestas de solución generadas a partir de los mismos, son un factor clave para lograr que los profesores y estudiantes de la comunidad académica de la Universidad acepten y adopten los productos y servicios que se tengan como resultados de este ciclo. Realizar una propuesta de difusión acertada y hallar los canales adecuados para tal fin hacen parte de las prioridades de este proyecto para cumplir con éxito el objetivo planteado.

Encontramos, también, que los hallazgos determinados a través de la VT se relacionan con lo planteado por Padilla y Valdivia (2015), quienes sostienen que el uso de bases de datos, plataformas virtuales y repositorios, constituyen elementos centrales para fortalecer y fomentar la producción científica en las Instituciones Educativas Superiores. En este orden de ideas, proponer soluciones que atiendan las necesidades de docentes y estudiantes en estos aspectos, resulta indispensable para mejorar la calidad académica y favorecer la innovación.

\section{Referencias}

Aldera, A. S. (2015). Investigating Multimedia Strategies to Aid L2 Listening Comprehension in EFL Environment. Theory and Practice in Language Studies, 5 (10), 1983-1988. Recuperado de: http:// www.academypublication.com/ojs/index.php/ tpls/article/view/tpls051019831988/413

Aldasoro-Alustiza, J.C., Cantonnet-Jordi, M.L., \& CiIleruelo-Carrasco, E. (2012). La vigilancia tecnológica y la inteligencia competitiva en los estándares de gestión de la calidad en I+D+i. XVI Congreso de ingeniería de organización, 1162-1168. Recuperado de: http://adingor.es/congresos/web/uploads/ cio/cio2012/SP_04_Gestion_Innovacion_Tecnologica_y_Organizativa/1162-1168.pdf 
Asociación Española de Normalización \& Certificación. (2011). Norma Española Experimental UNE 166006 Gestión de la I+D+i: Sistema de Vigilancia Tecnológica. Madrid, España: AENOR.

Baker, P., Rodríguez-Duarte, A., \& Sandulli, F. (2014). Un marco de análisis para la adopción de Sistemas de Gestión de Aprendizaje en las Universidades. Revista de Globalización, Competitividad y Gobernabilidad, 8 (1), 124-140. Recuperado de: https:// gcg.universia.net/article/view/466/marco-analisis-adopcion-sistemas-gestion-aprendizaje-universidades-

Bates, T. (2010). New challenges for universities: Why they must change. En UD, Ehlers \& D. Schneckenberg (eds.), Changing cultures in higher education: Moving Ahead to Future Learning, 15-25. Heidelberg, Alemania: Springer.

Bautista, G., Borges, F., \& Forés, A. (2006). Didáctica universitaria en entornos virtuales de enseñanza-aprendizaje. Madrid, España: Narcea.

Bollás, R., \&Valencia, L. (2017). Análisis de los modelos de la Vigilancia Tecnológica e inteligencia competitiva en proyectos de I+D+i. En XVII Congreso de la Asociación Latino-lberoamericana de Gestión Tecnológica-ALTEC 2017. Recuperado de: http://www. uam.mx/altec2017/pdfs/ALTEC_2017_paper_323. pdf

Casas, M., \& Stojanovic, L. (2013). Innovation in Ibero-American Universities. Revista De Universidad $Y$ Sociedad Del Conocimiento (RUSC), 10 (1), 240-253. Recuperado de https://link.springer.com/article/10.7238/rusc.v10i1.1345.

Castells, M. (2002). La era de la información (tomo I). México: Siglo XXI Editores.

Ferro, J. (2014). Retos de la educación superior en Colombia. En A. Roa y I. Pacheco (eds.), Educación superior en Colombia. Doce propuestas para la próxima década, 63-88. Barranquilla, Colombia: Editorial Universidad del Norte.

Gros-Salvat, B., \& Lara-Navarra, P. (2009). Estrategias de innovación en la educación superior: el caso de la Universitat Oberta de Catalunya. Revista Iberoamericana de Educación, (49), 223-245. Recuperado de: https://rieoei.org/RIE/article/view/681

Infante-Moro, A., Torres, J.C., Infante-Moro, J.C., \& Muñoz, M. (2014). Evaluación del uso de las bases de datos electrónicas en la docencia universitaria de la Universidad de Huelva. RED, Revista de Educación a Distancia, 40, Recuperado de: https://www. um.es/ead/red/40/infante.pdf

Ferrés, J., Masanet, M. J., \& Mateus, J. C. (2018). Three paradoxes in the approach to educational technology in the education studies of the Spanish universities. International Journal of Educational Technology in Higher Education, 15 (1), 15. Recuperado de: https://link.springer.com/article/10.1186/ s41239-018-0097-y

Harmer, J. (2003). The Practice of English Language Teaching (3rd ed.). Malaysia: Longman.

Marulanda, C. E., Hernández, A., \& López, M. (2016). Vigilancia Tecnológica para Estudiantes Universitarios: El Caso de la Universidad Nacional de Colombia, Sede Manizales. Formación universitaria, 9 (2), 17-28. Recuperado de: https://scielo. conicyt.cl/pdf/formuniv/v9n2/art03.pdf

Matus, G, Menay, P., Loyola, E., \& Cordeiro, I. (2009). Las bases de datos van a clases. XIV Conferencia Internacional de Bibliotecología" Información y ciudadanía: desafíos públicos y privados. Recuperado de: http://www.bibliotecarios.cl/descargas/2009/10/ matus_et_al.pdf

Ministerio de Educación Nacional (2016). Primer informe de indicadores de uso educativo de las tecnologías de la información y la comunicación - TIC, a partir de la aplicación, recolección, procesamiento y análisis de información de fuentes primarias y se- 
cundarias. Observatorio Colombiano de Innovación Educativa con uso de TIC. Proyecto desarrollado en el marco del contrato Interadministrativo No. 0739 de 2016 suscrito entre el Ministerio de Educación Nacional y la Universidad del Valle.

Moya-Espinosa, P., \& Moscoso-Durán, F. (2017). Vigilancia tecnológica e inteligencia competitiva en el modelo empresarial del sector hotelero colombiano. Revista De Investigación, Desarrollo E Innovación, 8 (1), 11-22. doi: https://doi. org/10.19053/20278306.v8.n1.2017.7367

Muchlis, M. (2015). The Implementation of Technology Approaches to Develop Learners' Autonomy in Learning English. Al-Ta'lim Journal, 22 (3), 276285. Recuperado de: https://www.researchgate. net/publication/287506341_The_Implementation_of_Technology_Approaches_to_Develop_ Learners'_Autonomy_in_Learning_English

Padilla, W., \& Valdivia, F. (2015). El impacto de las TIC en la universidad del siglo XXI. En E. Chinkes (coord.), Las Tecnologías de la Información y la Comunicación Potenciando la Universidad del Siglo $X X I, 19-26$. RedCLARA. Recuperado de: http://tical2015.redclara.net/images/docs/LasTICPotenciandolaUniversidadDeISXXI-TICAL2015.pdf

Rogers, E. M. (1995). Diffusion of Innovations (4th ed.). New York: The Free Press.

Salinas, J. (2004). Innovación docente y uso de las TIC en la enseñanza universitaria. RUSC. Universities and Knowledge Society Journal, 1 (1), 1-16. doi: http://dx.doi.org/10.7238/rusc.v1i1.228

Salmon, G. (2010). Learning innovation for the twenty-first century. En UD, Ehlers \& D. Schneckenberg (eds.), Changing cultures in higher education: Moving Ahead to Future Learning, 27-41. Heidelberg: Springer.

Torello, O. (2011). El profesor universitario sus competencias y formación. Profesorado. Revista de curriculum y formación del profesorado, 15 (3), 195 -
211. Recuperado de: http://www.ugr.es/ recfpro/ rev153COL1.pdf

Universidad del Valle (2012). Plan de incorporación de TIC a los procesos educativos 2012-2025. Cali, CoIombia: Univalle.

Universidad del Valle (2015-2018). Dirección de Nuevas Tecnologías y Educación Virtual DINTEVQuiénes somos. Recuperado de: http://dintev.univalle.edu.co/quienes-somos

Vandergrift, L. (2007). Recent developments in second and foreign language listening comprehension research. Language teaching, 40 (3), 191-210. Recuperado: https://www.cambridge.org/core/ journals/language-teaching/article/recent-developments-in-second-and-foreign-language-listening-comprehension-research/59FCACBDE27E3B2CEFA26A8182489FFB

Villegas, L. (2010). Formación de los docentes universitarios campo de análisis- Universidad de Caldas (Tesis doctoral). Universidad del Valle. Cali, Colombia. Recuperado de http://hdl.handle. net/10893/8305 
